\title{
Estimation of Bulk Density Distribution in Particle Charging Process Using Discrete Element Method Considering Particle Shape
}

\author{
Masatoshi AKASHI, ${ }^{1)}$ Hiroshi MIO, ${ }^{2)}$ Atsuko SHIMOSAKA, ${ }^{11}$ Yoshiyuki SHIRAKAWA, ${ }^{1)}$ Jusuke HIDAKA ${ }^{11}$ and \\ Seiji NOMURA ${ }^{3)}$
}

1) Department of Chemical Engineering and Materials Science, Doshisha University, 1-3 Tatara-Miyakodani, Kyotanabe, Kyoto 610-0321 Japan. $\quad$ 2) Kyoto Fine Particle Technology, Keihanna Interaction Plaza Inc., 1-7 Hikaridai, Seika-cho, Sorakugun, Kyoto 619-0237 Japan. $\quad 3$ 3) Environment \& Process Technology Center, Nippon Steel Corporation, 20-1 Shintomi,

Futtsu, Chiba 293-8511 Japan.

(Received on May 9, 2008; accepted on July 29, 2008)

\begin{abstract}
In order to analyze the segregation of coal particles in coke oven, it is necessity to consider the coal shape in Discrete Element Method (DEM). In this paper, the effect of particle shape on the granular flow and on bulk density distribution was investigated, and the novel method for considering particle shape on the void fraction in packed bed was proposed by changing contact distance between particles. The flowing behavior of tablet shaped polystyrene (PS) particles was analyzed and compared with simulation result which was calculated by spherical element or multi-sphere element. The results of flowing velocity and its angle using spherical element and multi-sphere one gave the similar tendency and they were correlated with experimental ones. Thus, the flowing behavior of non-spherical particle can be simulated by spherical element, as long as the suitable parameters were used. The bulk density distribution in horizontal direction in the simulated results using spherical element gave the different tendency of experimental one. On the other hand, those in multi-sphere element and new method showed the similar tendency to the experimental one. Thus, the bulk distribution cannot be simulated by using spherical element. The calculation speed of new method is 4.5 times faster than that of multi-sphere method. This method could be applied to coal packing simulation, and the bulk density distribution by using it had a good correlation with experimental one. Therefore, this method for considering the particle shape on the charged bed is very useful.
\end{abstract}

KEY WORDS: bulk density; coal; DEM; variable particle element method; particle shape.

\section{Introduction}

When granular materials are flowing, the particle segregation always occurs by the differences of particle characteristics, e.g., particle size, density and shape. Unfortunately, this phenomenon leads to serious problems in many particulate processes such as packing, conveying, mixing and so on. One of the most important issues in coke production process of iron-making is to understand and to control the bulk density distribution in packed coal layer caused by the particle size segregation, because the quality of cokes is affected by the bulk density distribution during carbonizing. ${ }^{1)}$ Recently, an experiment on coal charging using $1 / 8$ scale model of coke oven was tried to evaluate the bulk density distribution in coke oven ${ }^{2}$ and a mathematical model for coke oven charging has been reported. ${ }^{3)}$ However, it is still difficult to estimate the bulk density distribution under any conditions, because there are many parameters for operating coke oven, such as charging condition, particle size distribution, moisture content, and so on. The computational simulation using Discrete Element Method $(\mathrm{DEM})^{4)}$ is very effective for grasp of individual granular behavior and estimation of bulk density distribution. However it has a huge calculation load, so that, the granular materials are generally assumed to be spheres due to ease of contact detection between particles. Thus in order to analyze the particle segregation of coal particles in coke oven, it is necessity to consider the particle shape in DEM, because coal particles have complicated shape. There are mainly two effects of particle shape on granular behavior; i.e. particle rebounding angle and void fraction in packed bed. The particle shape affects rebounding angle if a particle impacts a wall. However, it might not affect during flowing, because there are many particles in the flow, each particle can not rebound individual way. Thus, the granular flow of the irregular particles will be similar to the one of spherical particles. Therefore, it is thought that the effect of particle shape on the void fraction will be more important during packing in coke oven.

Multi-sphere element method is one of the most wellknown methods for considering particle shape in DEM. ${ }^{5-8)}$ This method has an advantage for being able to represent particles having various shapes by bonded multiple spherical elements. The main feature of this method is representa- 
tion of non-spherical particle with high accuracy. However, in order to represent complicated shapes, many spherical elements are required for a particle, and the calculation speed becomes extremely slow. Thus, it doesn't really suit to the large scale calculation. The scale of coke oven is huge and there are uncountable particles in it. Therefore, the novel method for considering particle shape having low calculation load is necessary to analyze the particle segregation in coke oven.

In this paper, the effect of particle shape on the granular flow was investigated by using multi-sphere element particle or spherical one. The novel method (variable particle element method) for considering particle shape on the void fraction in packed bed was proposed by changing contact distance between particles which was related to particle circularity, and the bulk density distributions of tablet shaped polystyrene particle or coal in packed bed were analyzed.

\section{Experimental}

\subsection{Effect of Particle Shape on Flowing Behavior}

Effect of particle shape on flowing behavior was observed by using high speed video recorder (MotionPro X4, REDLAKE). The tablet shaped polystyrene (PS) particles were dropped on an oblique plate having 30 degree against the horizontal direction (Fig. 1). The diameter and thickness of tablet shaped PS particle are $3.4 \mathrm{~mm}$ and $2.0 \mathrm{~mm}$, respectively. The velocity and the direction of flowing particles were measured by Particle Image Velocimetry (PIV) using the software (Dipp-Flow ver.1.21, Ditect Corporation), and compared with simulated results.

\subsection{Effect of Particle Shape on Bulk Density Distribu- tion in Charged Bed}

The coal or PS particles were charged in containers to investigate the effect of particle shape on the bulk density distribution in charged bed. The tablet shaped PS particle, and irregular shaped coal particles, which were sieved by $2.83 \mathrm{~mm}$ and $2.00 \mathrm{~mm}$ meshes, were used in this work. The sizes of containers were H $150 \mathrm{~mm} \times W 70 \mathrm{~mm} \times \mathrm{D} 20 \mathrm{~mm}$ for coal and H $225 \mathrm{~mm} \times \mathrm{W} 105 \mathrm{~mm} \times \mathrm{D} 30 \mathrm{~mm}$ for PS, and the particles were charged into them by using a hopper. The bulk density distribution in charged bed was obtained by using X-ray computed tomography scanner (Subrina, SCT7800CT, Shimadzu Corporation). The Hounsfield number of each area was measured, and the bulk density of measured area was estimated by using the relation between Hounsfield number and bulk density (Fig. 2). The Houns-

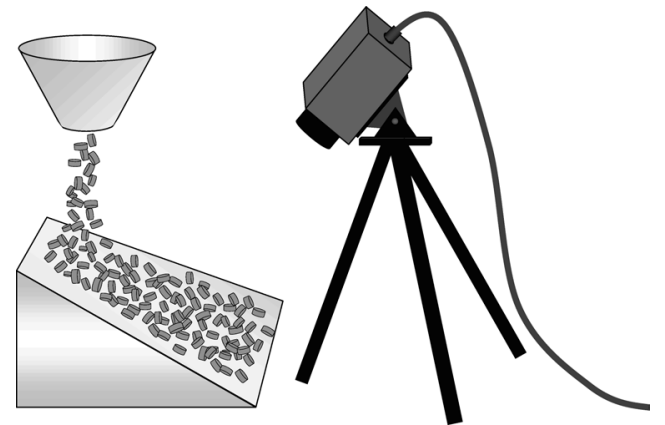

Fig. 1. Schematic diagram of experimental apparatus for recording particle flow. field number is calculated by difference of X-ray absorption efficiency of scanned object, as given by Eq. (1).

$$
\text { Housefield number }=\frac{\mu_{\mathrm{t}}-\mu_{\mathrm{w}}}{\mu_{\mathrm{w}}} \times 1000
$$

Where, $\mu_{\mathrm{t}}$ and $\mu_{\mathrm{w}}$ are X-ray absorption coefficient of scanned object and the water, respectively. Figure 2 was obtained by measuring Hounsfield number for small container which was filled with coal or PS particles, changing the tapping condition.

\section{Simulation}

\subsection{Discrete Element Method}

Discrete element method (DEM) is the simulation method for analyzing particle behavior by solving the Newton's second low to each particle. The particles during flowing collide and/or shear with nearby particles continuously, therefore, the calculation of contact force between particles is very important. Voigt model (Fig. 3), which consists

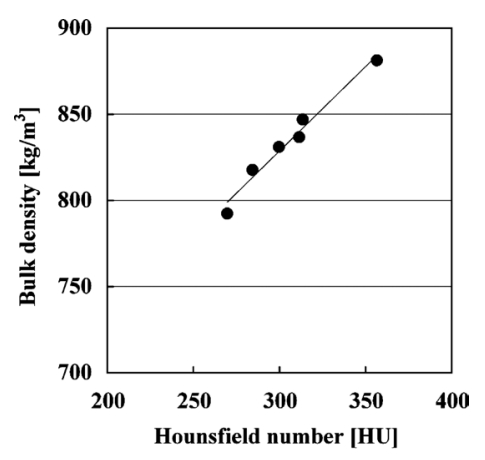

(a) Polystyrene particle

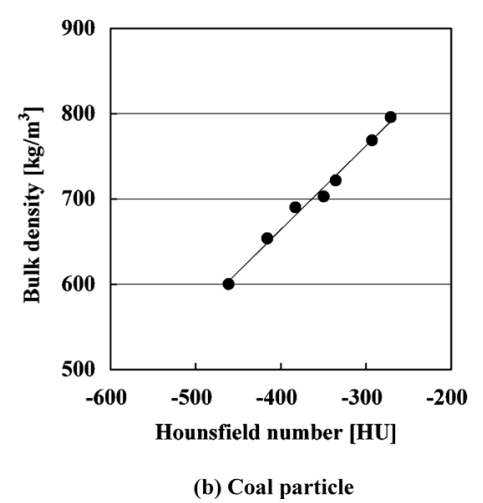

Fig. 2. Relation between bulk density and Hounsfield number.
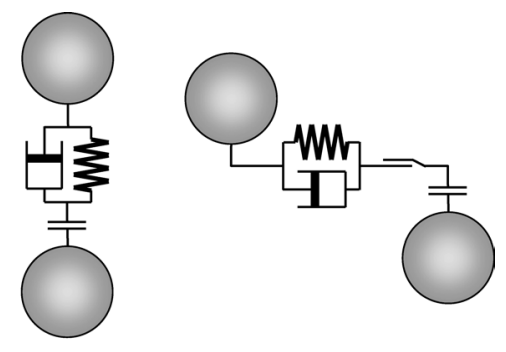
(a) Compressive
(b) Shear force

Fig. 3. Calculation model of contact force between particles. 
elastic spring and viscous dashpot, is usually used for the calculation of contact force in DEM. The effect of friction between particles is considered by a slider into shear direction. The elastic nonlinear spring based on Heltz and Mindlin contact theory as described in Eqs. (2)-(5) ${ }^{9-11)}$ is used in this simulation. The viscous dashpot coefficient, $\eta$, is defined by Eqs. (6), (7). Subscripts $n$ and $s$ denote normal and shear components.

$$
\begin{aligned}
& K_{\mathrm{n}}=\frac{4 b}{3 \pi}\left(\frac{1}{\delta_{i}+\delta_{j}}\right) \\
& \delta_{i}=\frac{1-v_{i}^{2}}{\pi \cdot E_{i}} \\
& K_{\mathrm{s}}=8 b\left(\frac{2-v_{i}}{G_{i}}+\frac{2-v_{j}}{G_{j}}\right) \\
& G_{i}=\frac{E_{i}}{2\left(1+v_{i}\right)} \\
& \eta_{\mathrm{n}}=2 \gamma \sqrt{m K_{\mathrm{n}}} \\
& \eta_{\mathrm{s}}=2 \gamma \sqrt{m K_{\mathrm{s}}}
\end{aligned}
$$

Where $E$ and $v$ are Young's modulus and Poisson's ratio and $b$ means the radius of contact face, $m$ and $\gamma$ are mass of a particle and coefficient of restitution, respectively.

\subsection{Multi-sphere Element Method}

Multi-sphere element method is commonly used when non-spherical particles are simulated in DEM. Multiple spherical elements are combined together to make an irregular-shaped body as shown in Fig. 4. The contact detection between bodies is executed by searching each spherical element which makes irregular bodies, and the contact force is also calculated between spherical elements. The moment of force at each contact is calculated by considering the distance between the center of gravity and contact point. This method has an advantage of being able to model the complicated shape, however, total number of particles increases significantly with increasing constitutive particles.

\subsection{Variable Particle Element Method}

The shape of coal particle is extremely irregular and this shape makes the void in the charged bed, as shown in Fig. 5. The contact distance between particles is not constant when the irregular-shaped particles are in contact. Thus, it should be considered in the contact detection process in DEM. The new method, named variable particle element method, for considering the effect of particle shape on the contact detection is based on the spherical element due to less calculation load. The distance between the center of gravity and particle surface is given by $R_{\min , i} \leq R_{i} \leq R_{\max , i}$, and the contact distance also depends on $R_{i}$. Therefore, the contact distance, $r_{\mathrm{c}, i}$, is changed randomly from $R_{\min , i}$ to $R_{\max , i}$ at each contact. $R_{\max , i}$ and $R_{\min , i}$ should be related to the particle shape, and they are evaluated by circularity of

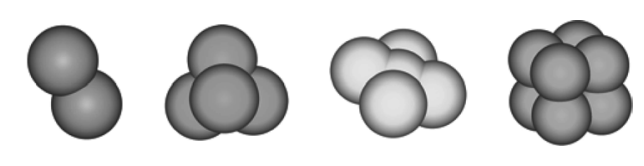

Fig. 4. Examples of irregular-shaped particles using multisphere element method.

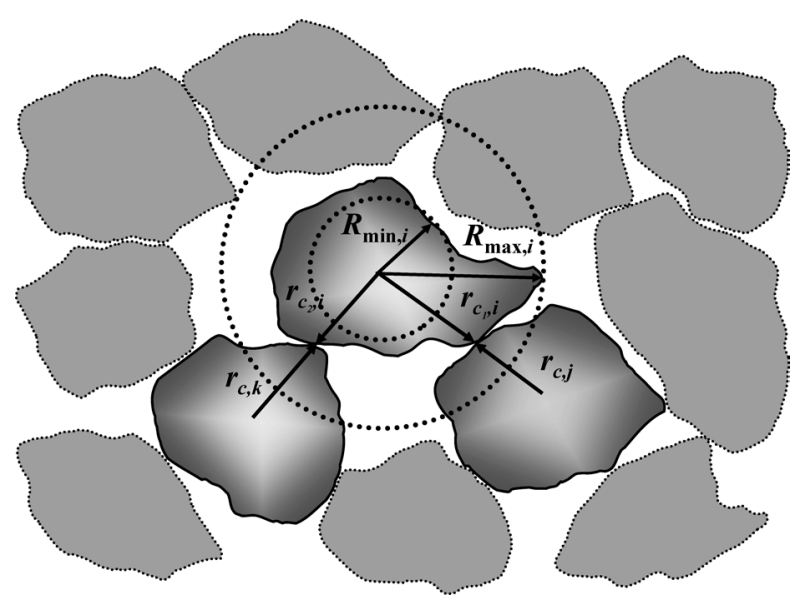

Fig. 5. Void distribution in charged coal bed.

coal particle. The calculation procedure is as follows.

When two particles are coming closer, the distance between centers of particles, $d_{i, j}$, is calculated. If $d_{i, j} \leq R_{\max , i}+$ $R_{\text {max }, j}$, these particles are considered to have a possibility of contact. Then, the distances between the particle center and contact point of this particle pair, $r_{\mathrm{c}, i}$ and $r_{\mathrm{c}, j}$, are given by generating random numbers. Where $r_{\mathrm{c}, i}$ and $r_{\mathrm{c}, j}$ are satisfied with Eqs. (8), (9)

$$
\begin{aligned}
& R_{\min , i} \leq r_{\mathrm{c}, i} \leq R_{\max , i} \\
& R_{\min , j} \leq r_{\mathrm{c}, j} \leq R_{\max , j}
\end{aligned}
$$

The contact detection is executed by using $r_{\mathrm{c}, i}$ and $r_{\mathrm{c}, j}$, i.e., $d_{i, j}<r_{\mathrm{c}, i}+r_{\mathrm{c}, j}$, is the contacting condition. Once $r_{\mathrm{c}, i}$ and $r_{\mathrm{c}, j}$ are given, these values are kept until the distance between these particles becomes larger than $R_{\max , i}+R_{\max , j}$. The physical properties of the particles which are used in the force calculation are assumed as the values of volume mean particle size. $r_{\mathrm{c}, i}$ and $r_{\mathrm{c}, j}$ are generated on every particle pair at the first approach, and they are deleted after two particles go away. The calculation flow diagram is shown in Fig. 6. The maximum contact distance, $r_{\mathrm{c}, \max }$, and the minimum contact distance, $r_{\mathrm{c}, \min }$, of PS particle can be estimated easily because of its tablet shape. The contact distance for the tablet shaped PS particle and the frequency of each contact distance were given in Table 1.

On the other hand, it is difficult to get the contact distance between coal particles due to having individual irregular shape. Thus, the shape distribution of coal particle was estimated by the circularity, $\varphi$, which is given by Eq. (10).

$$
\varphi=\frac{4 \pi \times S}{A^{2}}
$$

Where, $S$ and $A$ are the projected area of particle and the surrounding length, respectively. The projected area, the surrounding length and the distance between center of gravity and particle surface of coal particles were measured by 


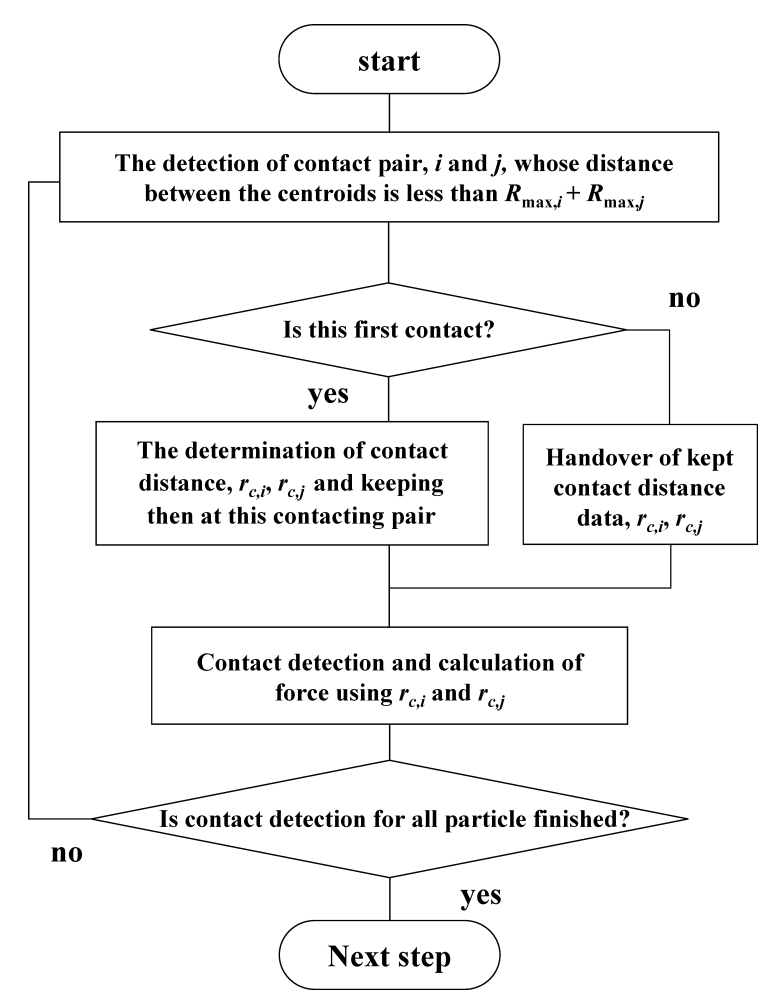

Fig. 6. Program flowchart of variable particle element method.

Table 1. Frequency of contact distance of tablet shaped polystyrene particle.

\begin{tabular}{cc}
\hline$r_{c} / r_{c, \text { mean }}$ & Frequency $[\%]$ \\
\hline 0.613 & 0.43 \\
0.637 & 3.50 \\
0.705 & 7.00 \\
0.805 & 10.49 \\
0.927 & 13.99 \\
1.064 & 17.49 \\
1.210 & 16.23 \\
1.120 & 12.35 \\
1.063 & 12.35 \\
1.043 & 6.17 \\
\hline
\end{tabular}

using the software (WinRoof ver.5.5, Mitani Corporation), and its distribution of 1000 coal particles are shown in Fig. 7. The relation between $\varphi$ and $r_{\mathrm{c}, \text { max }}$ or $r_{\mathrm{c}, \min }$ are shown in Table 2. Each particle has different $\varphi$, and the contact distance was determined randomly according to $\varphi$.

\subsection{Effect of Particle Shape on Flowing Behavior}

Effect of particle shape on flowing behavior was simulated by using tablet shaped PS particles, which were modeled by spherical element or multi-sphere element method. The diameter of spherical PS particle was determined to be mean volume diameter $(3.26 \mathrm{~mm})$ and the PS particle had a larger rolling friction force than that of normal spherical particle to simulate the non-spherical particles behavior. Under the multi-sphere element method, 18 spherical elements having $1.3 \mathrm{~mm}$ in diameter were used for a PS particle, which is shown in Fig. 8. These particles were fallen to an oblique plate having 30 degree against the horizontal

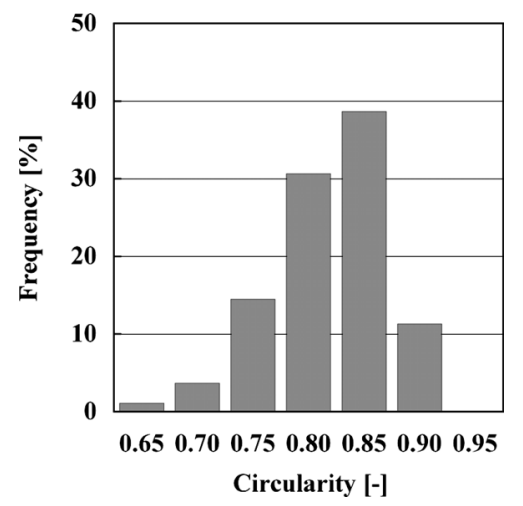

Fig. 7. Distribution of circularity under the condition of 2.00-2.83 $\mathrm{mm}$ particles.

Table 2. Range of contact distance for each circularity of coal particle.

\begin{tabular}{ccc}
\hline$\varphi$ & $r_{c, \max } / r_{c, \text { mean }}$ & $r_{c, \min } / r_{c, \text { mean }}$ \\
\hline 0.65 & 1.498 & 0.544 \\
0.70 & 1.463 & 0.597 \\
0.75 & 1.379 & 0.658 \\
0.80 & 1.317 & 0.718 \\
0.85 & 1.248 & 0.775 \\
0.90 & 1.119 & 0.833 \\
\hline
\end{tabular}

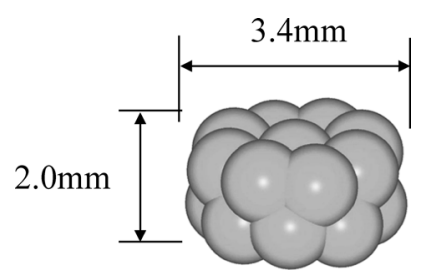

Fig. 8. Tablet shaped polystyrene particle using multi-sphere element method.

using a hopper, and the velocity field and flowing direction were measured by PIV, and they were compared with experimental results.

\subsection{Particle Packing Simulation}

PS and coal particles were charged into the container from the hopper to investigate the effect of particle shape on bulk density distribution. The sizes of the containers were the same as those in experimental. For the case of PS particles, the multi-sphere element, spherical element or variable particle element method were used and compared with those methods. The coal particles from 2.00 to $2.83 \mathrm{~mm}$ having Rosin-Rammler distribution were calculated in the simulation using the spherical element or variable particle element method. The calculation conditions are tabulated in Table 3, and Young's modulus was 1000 times smaller than actual value to reduce the calculation time. The effect of this adjustment on the calculation result was already checked.

\section{Results and Discussion}

\subsection{Particle Flowing Behavior}

Figure 9 shows the image of flowing PS particles in the 
Table 3. Calculation condition in simulation work.

\begin{tabular}{lccc}
\hline & & polystyrene & coal \\
\hline Time step [s] & & $1 \times 10^{-6}$ & $1 \times 10^{-6}$ \\
Density of particle $\left[\mathrm{kg} / \mathrm{m}^{3}\right]$ & 1252 & 1301 \\
Poisson's ratio [-] & Particle & 0.35 & 0.35 \\
& Wall & 0.25 & 0.25 \\
Young's modulus & Particle [MPa] & 1.39 & 4.5 \\
& Wall [GPa] & 3.4 & 3.4 \\
Coefficient & Particle-particle & 0.210 & 0.693 \\
$\begin{array}{c}\text { of restitution [-] } \\
\text { Coefficient } \\
\text { of friction [-] }\end{array}$ & Wall-particle & 0.171 & 0.779 \\
& Particle-particle & 0.53 & 0.39 \\
$\begin{array}{c}\text { Coefficient of } \\
\text { rolling friction [-] }\end{array}$ & Particle-particle & 1.33 & 5.85 \\
& Wall-particle & 3.15 & 4.00 \\
\hline
\end{tabular}

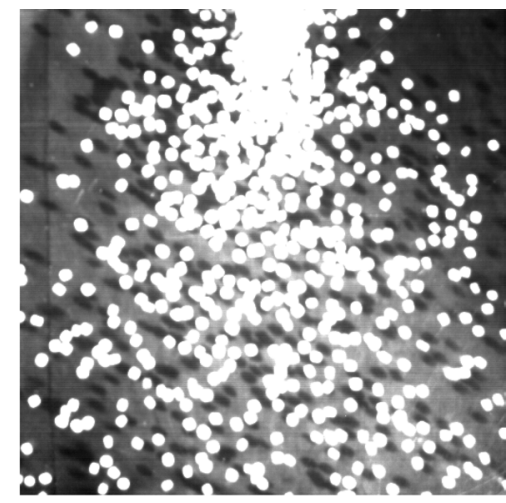

(a) Experimental

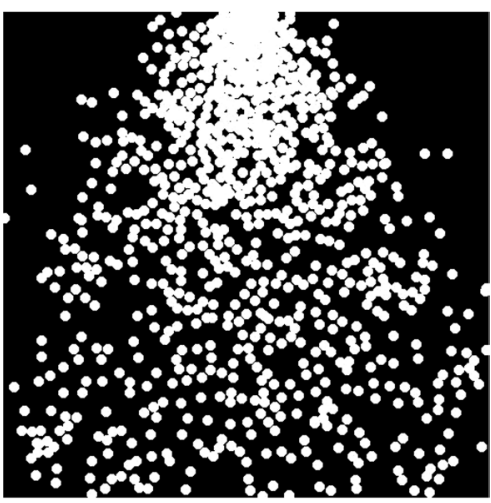

(b) Simulated by spherical element method

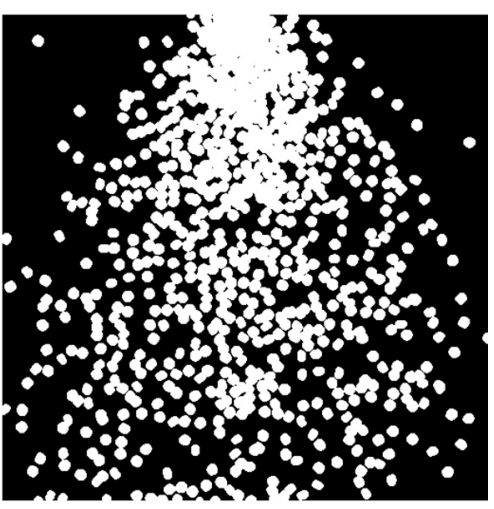

(c) Simulated by multi-sphere element method

Fig. 9. Snapshots of flowing polystyrene particles.



Fig. 10. Relation between mean horizontal velocity and particle position.

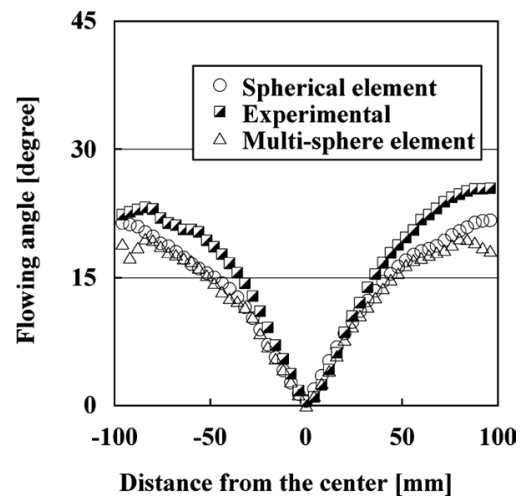

Fig. 11. Relation between mean flowing angle and particle position.

experimental work and the snapshots of simulated ones. The image of experiment shows that PS particles dropped from the hopper spread on the plate. The snapshots of spherical element and multi-sphere element in the simulation works also show similar behavior. Figure $\mathbf{1 0}$ shows the relation between mean particle velocity during flowing and the particle position. The mean velocity of experimental result decreases little with increasing the distance from the center. This tendency is also seen in both simulated results and the most of the values are quite similar. Figure 11 shows the relation between mean flowing angle of particle and particle position. The flowing angle against the vertical direction increases with increasing the distance from the center of flow due to the spread of flowing particles. Simulated results using spherical or multi-sphere element are compatible with the experimental result according to this analysis. Thus, it is concluded that the effect of particle shape on flowing behavior is little, if the particles are flowing with many particles and the suitable parameters, such as the rolling friction, are set in the calculation. Therefore, the spherical element can be used for the simulation of nonspherical particle flow.

\subsection{The Bulk Density Distribution of PS Particles}

Figure 12 shows the X-ray image of PS particle bed in the experimental work and the snapshots of simulated ones. The angles of repose of experiment, spherical element, multi-sphere element and variable particle element method are 25 degree, 33 degree, 28 degree and 27 degree, respectively. These results indicate that particle charging behavior 


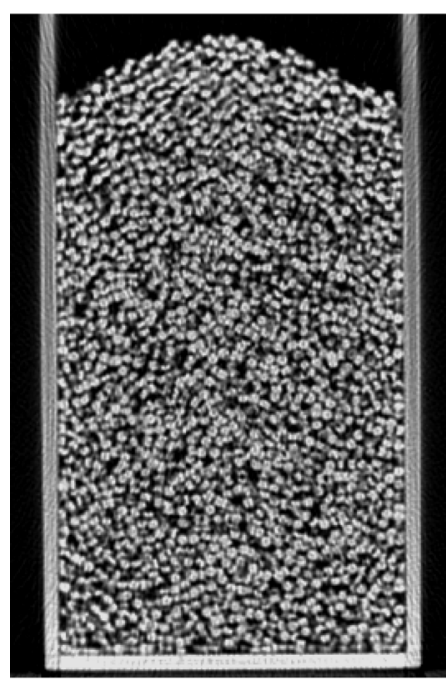

(a) Experimental

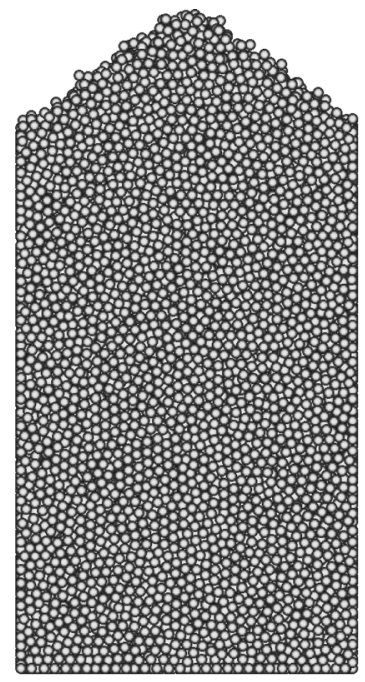

(b) Snapshot of spherical element method

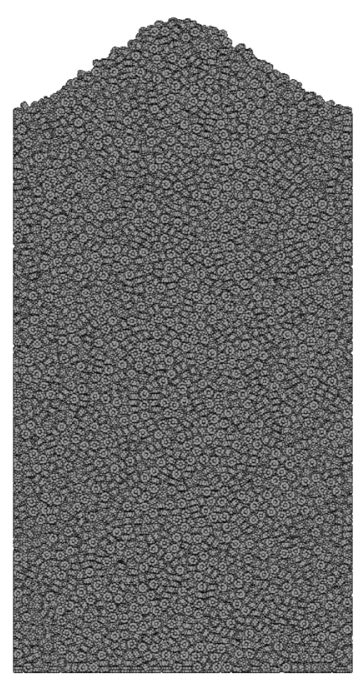

(c) Snapshot of multi-sphere element method

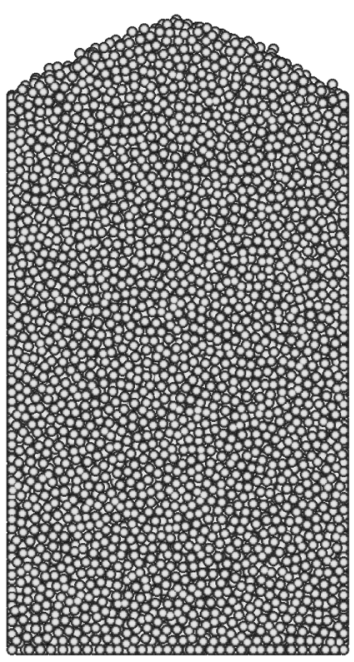

(d) Snapshot of variable particle element method

Fig. 12. Images of packed polystyrene particles in container.

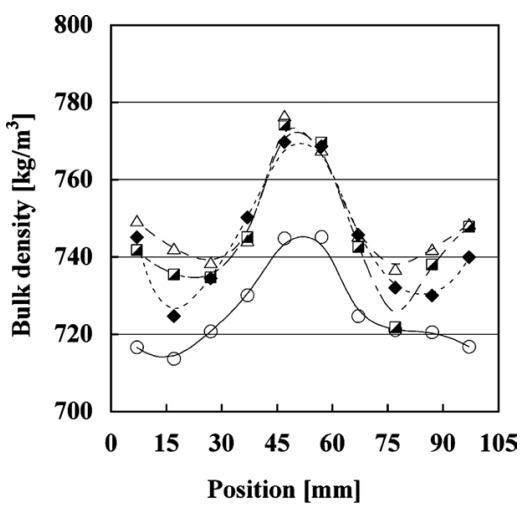

- Spherical element
- Experimental
$\triangle-$ Multi-sphere element
- Variable particle element

Fig. 13. Bulk density distribution of polystyrene particles in horizontal direction.

of each calculation method is a little different, and the heap of the simulation using the spherical element is different from that of experimental.

Figure 13 shows bulk density distribution in the container in horizontal direction until $140 \mathrm{~mm}$ in height for experimental and simulated results. The bulk density in the experimental work was obtained by Hounsfield number of each area, and the one in the simulation was given by calculating the volume fraction of each grid. The experimental result shows that the bulk density at the center is the largest and it decreases with increasing the distance from the center because the particles around the charging area have large compressive force. The tablet shaped particle is also easily packed along with the side wall, so that the bulk density near side wall increases. Most of simulated results have similar tendency to experimental one, however the result using spherical element is smaller than experimental result and the bulk density near side wall shows the different tendency. Thus, the simulation using the spherical element cannot give actual phenomenon of bulk density distribution in charged bed because the particle shape affects the void

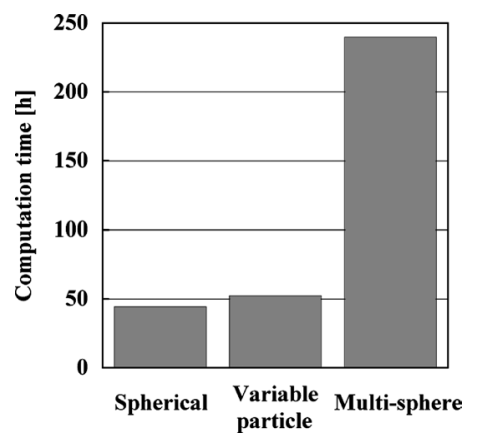

Fig. 14. Computation time for packing simulation.

fraction. The simulation results using multi-sphere element and variable particle element are compatible with experimental result, and good agreements are seen in both results. Therefore, both methods can be used for the analysis of bulk density distribution in charged bed under the nonspherical particles.

Figure 14 shows the calculation times for three methods. The calculation time of multi-sphere element method is 5.4 times larger than that of spherical element. Because 18 spheres make a tablet shaped body, and total number of spheres in the calculation is 360000 . On the other hand, the time of variable particle element is only 1.2 times larger than that of spherical element, and the additional calculation load is random number generation at every new contact. As explained above, the variable particle element method can reduce simulation time remarkably compared with the multi-sphere element method. Thus, it is concluded that this proposed method for considering particle shape is quite useful for estimating the bulk density distribution without huge calculation load.

\subsection{Bulk Density Distribution of Coal Particle}

Figure 15 shows the bulk density distribution of coal particles in horizontal direction until $98 \mathrm{~mm}$ in height. The bulk density at the center is the largest, and it decreases with increasing the distance from the center. Both simulated results give the similar tendency to experimental re- 


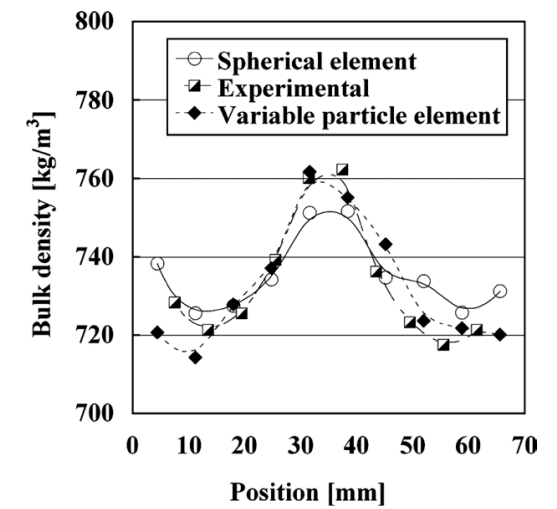

Fig. 15. Bulk density distribution of coal particle in horizontal direction.

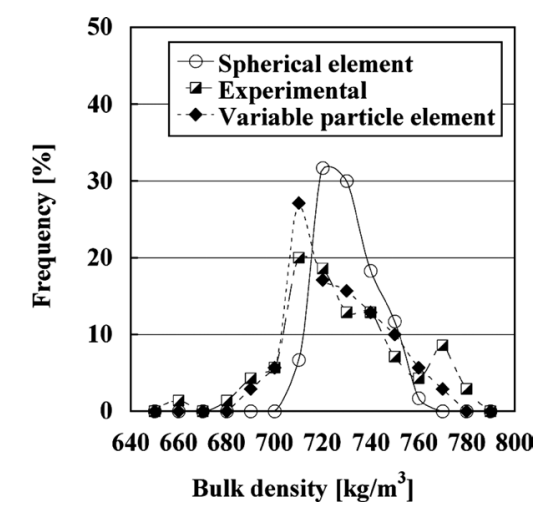

Fig. 16. Distribution of bulk density at each grid in coal particle bed.

sult, however, the difference between the maximum value and minimum one is much less than that of experimental when the spherical element was used in the calculation.

Figure 16 shows the distribution of bulk density at each grid in packed coal layer. The bulk densities of experimental and simulated by using variable particle element distributed widely and a good agreement between them is seen. On the other hand, the result calculated by using spherical element doesn't give an enough agreement with experimental result. Therefore, this new method is applicable to the estimation of the bulk density distribution in coke oven even if particles have significantly irregular shape.

\section{Conclusion}

The effect of particle shape on the granular flow was investigated by using spherical element or multi-sphere element method. The results indicate that the effect of particle shape on flowing behavior is little, and even spherical element can represent flowing behavior of non-spherical particles if the suitable parameters were used. The simulation of packing of tablet shaped PS particles was performed by using the multi-sphere element, spherical element or variable particle element method.

The bulk density distributions using multi-sphere element or variable particle element had good agreements with experimental result, on the other hand, the one of using spherical element didn't correlate with experimental one. Thus, the particle shape affected the bulk density distribution strongly because the simulations using multi-sphere element or variable particle element could represent the void fraction caused by the particle shape. In addition, the calculation speed of new method was 4.5 times faster than that of multi-sphere element method, thus this proposed method can consider the effect of particle shape on the bulk density distribution without huge calculation load. Finally, this method could be applied to coal packing simulation, and the bulk density distribution by using it had a good correlation with experimental one. Therefore, this new method is useful for the estimation of the bulk density distribution in coke oven even if particles have significantly irregular shape.

\section{REFERENCES}

1) S. Nomura, T. Arima and K. Kato: Fuel, 83 (2004), 1771.

2) S. S. Gupta, H. N. Prasad and H. S. Sandhu: Ironmaking Steelmaking, 28 (2001), 444.

3) J. L. Karst, E. Petit and J. P. Gaillet: Rev. Metall. Cah. Inf. Tech., 101 (2004), 447.

4) P. A. Cundall and O. D. L. Strack: Geotechnique, 29 (1979), 47.

5) E. Sakaguchi, S. Kawakami, S. Tamura and F. Tobita: J. JSAM, 58 (1996), 9 .

6) M. H. Abbaspour-Fard: Biosyst. Eng., 88 (2004), 153

7) F. Bertrand, L.-A. Leclaire and G. Lececque: Chem. Eng. Sci., 60 (2005), 2517.

8) Y. Song, R. Turton and F. Kayihan: Powder Technol., 161 (2006), 32.

9) K. Kaneta: Theory of Elasticity, Corona Corporation, Tokyo, (1973), 423.

10) B. R. D. Mindlin: J. Appl. Mech., 16 (1949), 259.

11) B. R. D. Mindlin and H. Deresiewicz: J. Appl. Mech., 20 (1953), 327. 